\title{
Meta-analysis of the prognostic and clinical value of tumor-associated macrophages in adult classical Hodgkin lymphoma
}

\author{
Baoping Guo, Hong Cen ${ }^{*}$, Xiaohong Tan and Qing Ke
}

\begin{abstract}
Background: The prognostic significance of tumor-associated macrophages (TAM) in adult classical Hodgkin lymphoma ( $\mathrm{CHL}$ ) remains controversial. Here, we report a meta-analysis of the association of CD68 and CD163 infiltration on the clinical outcome of adult $\mathrm{CHL}$.
\end{abstract}

Methods: A comprehensive search to identify relevant articles was performed in PubMed, Embase, and Google Scholar on January 31, 2016. Using the fixed effect or random effects model of DerSimonian and Laird, hazard ratios (HR) or odds ratios (OR) with $95 \%$ confidence intervals (Cls) were used as the effect size estimate.

Results: Twenty-two eligible studies with a total of 2959 patients were identified. Our analysis indicated that a high density of $\mathrm{CD}_{68}{ }^{+}$TAMs in the tumor microenvironment of adult $\mathrm{CHL}$ predicted poor overall survival (OS) (HR: 2.41; $95 \%$ Cl, 1.92-3.03), shorter progression-free survival (PFS) (HR: 1.78; $95 \% \mathrm{Cl}, 1.45-2.18$ ), and poor disease-specific survival (HR: 2.71; $95 \% \mathrm{Cl}$, 1.38-5.29). High density of CD163+ TAMs in the tumor microenvironment of adult CHL also predicted poor OS (HR: 2.75; $95 \% \mathrm{Cl}, 1.58-4.78$ ) and poor PFS (HR: 1.66; $95 \% \mathrm{Cl}, 1.22-2.27$ ). In addition, we demonstrated that a high density of either $\mathrm{CD}^{+} 8^{+}$or CD163 ${ }^{+}$TAMs was associated with the presence of Epstein-Barr virus in neoplastic cells (OR $95 \% \mathrm{Cl}, 2.02-4.84$; $\mathrm{OR}_{\mathrm{CD} 163}$ : 2.88; $\left.95 \% \mathrm{Cl}, 1.55-5.34\right)$. A high density of either $\mathrm{CD}^{+} 8^{+}$or $\mathrm{CD}_{163}{ }^{+} \mathrm{TAMs}$ tend to be associated with a more advanced clinical stage (OR $\mathrm{CD}_{68}: 1.25 ; 95 \% \mathrm{Cl}, 0.93-1.67$; $\mathrm{OR}_{\mathrm{CD} 163}$ : 1.19; 95 \% Cl, 0.86-1.63), B-symptoms (OR $\mathrm{CD}_{\mathrm{CD}}$ : 1.35; $95 \% \mathrm{Cl}$, 0.90-2.01; $\mathrm{OR}_{\mathrm{CD} 163}$ : 2.19; 95 \% Cl, 0.96-5.03), higher International Prognostic Factors Project Score (ORCD68: 1.20; $95 \%$ Cl, 0.67-2.15; OR $\mathrm{R}_{\mathrm{CD} 163}$ : 2.00; $95 \% \mathrm{Cl}, 0.92-4.35)$, and bulky disease (OR $\mathrm{CD}_{68}: 1.47 ; 95 \% \mathrm{Cl}, 0.88-2.47$; $\mathrm{OR}_{\mathrm{CD} 163}$ : 1.19; $\left.95 \% \mathrm{Cl}, 0.72-1.96\right)$.

Conclusions: Our analyses suggest that a high density of either $\mathrm{CD}^{+} 8^{+}$or $\mathrm{CD} 163^{+}$TAMs is a robust predictor of adverse outcomes in adult CHL. Increased TAMs should be taken into account to further improve prognostic stratification and the planning of appropriate therapeutic strategies.

Keywords: Tumor-associated macrophages, Classical Hodgkin lymphoma, Meta-analysis

\section{Background}

Classical Hodgkin lymphoma (cHL) carries an excellent prognosis for most patients, with more than $80 \%$ of patients experiencing long-term remission following conventional chemotherapy or radiotherapy-based protocols [1-3]. However, approximately $20 \%$ of patients experience relapse or disease that is refractory to all conventional therapies, and many patients may suffer

\footnotetext{
* Correspondence: cenhong01@163.com

Department of Chemotherapy, Affiliated Tumor Hospital of Guangxi Medical University, Nanning, People's Republic of China
}

short- and long-term treatment-related complications [4-6]. The International Prognostic Factors Project Score (IPS) is the most widely used prognostic system; however, it is rarely employed to modify treatment $[7,8]$. Robust prognostic indicators are thus needed to better risk-stratify patients at diagnosis.

cHL is unique among the lymphomas because malignant cells are heavily outnumbered by reactive cells in the tumor microenvironment, such as macrophages, $\mathrm{T}$ cells, B cells, eosinophils, mast cells, and other stromal elements. In an adult cHL microenvironment, malignant 
Hodgkin-Reed-Sternberg cells express a variety of cytokines and chemokines. This is thought to be the driving force behind an abnormal immune response, perpetuated by additional factors secreted by recruited reactive cells in the microenvironment [9]. Steidl et al. [10] were the first to show, by gene expression profiling followed by immunohistochemistry assay, that the quantity of tumor-associated macrophages (TAMs) predicts progression-free survival (PFS). However, most studies that used various antibodies to stain TAMs were inconclusive regarding the association of $\mathrm{CD}^{+} 8^{+}$and $\mathrm{CD}_{163}{ }^{+}$TAM density and survival. Some studies have indicated that a high density of either $\mathrm{CD} 68^{+}$or $\mathrm{CD} 163^{+}$ TAMs in the tumor microenvironment of adult $\mathrm{CHL}$ is associated with poorer outcomes [10-22]. Other studies have not confirmed this finding [23-31], and using TAMs as a biomarker to risk-stratify patients remains controversial.

The objective of this study was to evaluate the prognostic significance of elevated density of $\mathrm{CD} 68^{+}$and $\mathrm{CD}_{163}{ }^{+}$TAMs in the tumor microenvironment on overall survival (OS) and PFS in patients with adult cHL. In addition, the relationship between $\mathrm{CD} 8^{+}$and $\mathrm{CD} 163^{+}$ TAMs in the tumor microenvironment of adult $\mathrm{cHL}$ and other clinical characteristics was also examined.

\section{Methods}

The present meta-analysis was performed in accordance with the Preferred Reporting Items for Systematic Reviews and Meta-Analyses Statement [32].

\section{Literature search}

We performed a systematic electronic search in PubMed, Embase, and Google scholar for articles published before January 31, 2016. We identified studies by using Medical Subject Heading (MeSH) terms and corresponding keywords, including "macrophages", "tumorassociated macrophage", "tumor-infiltrating macrophage", "intratumoral macrophage", "TAMs", "Hodgkin disease", "classical Hodgkin lymphoma". No language restriction was applied. We also manually checked the bibliographies of previous reviews and references in all selected studies. Investigators were contacted and asked to supply additional data when key information relevant to the meta-analysis was missing.

\section{Selection criteria}

Studies were included if they (1) were prospective or retrospective cohort studies or clinical trials; (2) contained immunochemistry data used to evaluate TAM by antiCD68 or anti-CD163; (3) were studies that involved patients with a proven diagnosis of cHL performed to investigate the correlation of $\mathrm{CD}^{+} 8^{+}$and $\mathrm{CD} 163^{+}$ TAM density and survival; or (4) tumor-associated macrophages in adult cHL were described as high (above the cut-off value) and low (below the cut-off value) density. Studies were excluded if they were (1) review articles, case reports, animal or in vitro studies; or (2) analyzing pediatric patients or serum CD68 or CD163 samples.

\section{Study selection, data extraction, and end points}

Two investigators (BPG and HC) independently selected articles and extracted data from eligible studies. Disagreements were resolved by consensus. Baseline characteristics and outcomes were extracted from the selected articles. Information taken from each study included the name of the first author, year of publication, country, number of patients, stage, sex, treatment, WHO subtype, EpsteinBarr virus (EBV) status, antibodies, thresholds, median follow-up, and outcome correlation. In situ hybridization (ISH) analysis for EBV-encoded RNA (EBER) was performed in all included studies. Among the included studies, there were six studies using a follow-up time of less than 5 years $[13,16,23,26,27,29]$. We chose OS and PFS as endpoints for our meta-analysis. Various endpoints for PFS were reported in the selected studies, including event-free survival (EFS [12, 14, 18, 19, 22]) and failure-free survival (FFS) [15]. As PFS, EFS, and FFS had a similar definition in these articles, we operationally defined PFS to include EFS or FFS for studies that did not provide PFS.

\section{Quality assessment}

The Newcastle-Ottawa Scale (NOS) was used to assess the quality of each individual study; this was performed independently by two authors (BPG and XHT). The NOS comprises three quality parameters: selection (0-4 points), comparability ( $0-2$ points), and outcome assessment (0-3 points). Studies with NOS scores of $\geq 6$ were determined to be high-quality [33].

\section{Statistical analysis}

The primary outcome was survival in patients with a high density of either $\mathrm{CD}^{+} 8^{+}$or $\mathrm{CD} 163^{+}$TAMs compared to those with a low density of $\mathrm{CD}^{+} 8^{+}$or $\mathrm{CD} 163^{+}$ TAMs. The cut-off value for "high versus low" $\mathrm{CD} 68^{+}$or $\mathrm{CD}_{163}{ }^{+}$TAM density was determined by the investigators in each study. Hazard ratios (HRs) with $95 \%$ confidence intervals (CIs) were combined to obtain an effective value. For studies in which HRs and CIs were not available, we used the method proposed by Parmar et al. [34] to derive estimates from survival curves. An $\mathrm{HR}>1$ indicated poor survival in the group with high $\mathrm{CD} 8^{+}$or $\mathrm{CD} 163^{+}$TAM density.

For the pooled analysis of the relationship between high $\mathrm{CD} 8^{+}$or $\mathrm{CD} 163^{+}$TAM density and EBV status or other clinical parameters (such as stage), odds ratios 
(OR) and their $95 \%$ CIs were combined to give the effective value. An OR $>1$ indicated a higher probability that EBV was present and advanced stage in the group with high $\mathrm{CD} 8^{+}$or $\mathrm{CD} 163^{+}$TAM density. The point estimate of the HR or OR was considered statistically significant at the $P<0.05$ level if the $95 \% \mathrm{CI}$ did not include the value 1 . Heterogeneity was assessed by the $\mathrm{X}^{2}$ test and expressed as $I^{2}$ index [35], which describes the percentage of total variation across studies due to heterogeneity rather than chance $(25 \%$ low heterogeneity, $50 \%$ medium, $75 \%$ high). If heterogeneity existed between primary studies, a random effects model was used. Otherwise, a fixed effects model was used in metaanalysis [36]. If results of both univariate and multivariate Cox regression analyses were reported, multivariate models were used for a more accurate estimate of the effect of CD68 or CD163 expression. Begg's test [37] and Egger's test [38] were used to detect possible publication bias. All analyses were carried out using STATA statistical software package version 12.0 (STATA, College Station, TX).

\section{Results}

\section{Selection and characteristics of studies}

Our initial search yielded 1585 articles. After removing duplicates and screening the titles and abstracts, 31 articles were reviewed in further detail. After reviewing the full text, 22 unique studies were selected as potentially appropriate for inclusion in the meta-analysis [10-31]. Our search strategy is presented in Fig. 1.

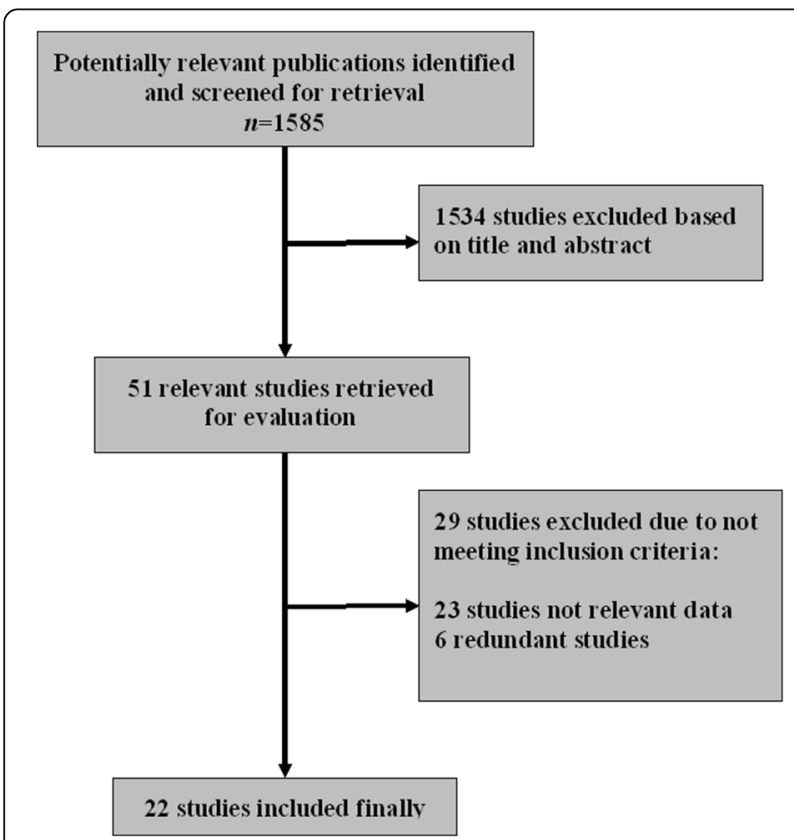

Fig. 1 Flow diagram of the systematic review and meta-analysis process
The main characteristics of the included studies are summarized in Table 1. Sixteen studies were retrospective cohorts and six were prospective cohorts. Studies were published between 2010 and 2016. The studies were conducted in 12 countries (Denmark, Italy, Switzerland, France, Spain, Israel, United Kingdom, Canada, United States, Japan, South Korea, Egypt, India, Serbia, and China). Population sizes ranged from 61 to 288 , with a total of 2959 patients. The reported median or mean age ranged from 21 to 54 years across eligible studies.

The points of study quality assessed on the NOS for assessing quality ranged from 3 to 9 (mean $=6.23$ ), with higher values indicating better methodology. Certainly, the low quality studies were also included in the analyses. The results of this quality assessment are shown in Table 2.

\section{Prognostic significance of $\mathrm{CD}^{+} 8^{+}$TAMs}

Fifteen studies were included in the analysis of $\mathrm{CD} 68^{+}$ TAMs on OS in adult cHL. The results of our metaanalysis showed that a high $\mathrm{CD}^{+} 8^{+}$TAM density was associated with shorter OS than a low CD68 ${ }^{+}$TAM density, with a pooled HR of 2.41 (95\% CI, 1.92-3.03). No significant heterogeneity was found across the studies $\left(I^{2}=12.7 \%, P=0.31\right)$. Twelve studies reported data on CD68 $8^{+}$TAMs and PFS in adult cHL. Meta-analyses results demonstrated that a high $\mathrm{CD} 68^{+}$TAM density was associated with shorter PFS than a low $\mathrm{CD}^{+} 8^{+} \mathrm{TAM}$ density, with a pooled HR of 1.78 (95\% CI, 1.45-2.18). No significant heterogeneity was found across the studies $\left(I^{2}=3.4 \%, P=0.41\right)$. HRs for disease-specific survival (DSS) were available in four studies. The estimated pooled HR showed that a high $\mathrm{CD} 68^{+}$TAM density was associated with poorer DSS than a low CD68 ${ }^{+}$TAM density, with a pooled HR of 2.71 (95\% CI, 1.38-5.29). No significant heterogeneity was found across the stu$\operatorname{dies}\left(I^{2}=0.0 \%, P=0.73\right.$; Fig. 2).

\section{Prognostic significance of $\mathrm{CD} 163^{+}$TAMs}

Meta-analysis of seven studies showed poorer OS in the high $\mathrm{CD}_{163^{+}}$TAM density group than in the low $\mathrm{CD}_{163^{+}}$TAM density group, with a pooled HR of 2.75 (95\% CI, 1.58-4.78). There was an indication of medium heterogeneity across the studies, but it did not reach statistical significance $\left(I^{2}=51.8 \%, P=0.053\right)$. HRs for PFS were available in five studies with adult classical Hodgkin lymphoma. The results of our meta-analysis showed that high $\mathrm{CD}_{163^{+}}$TAM density was associated with shorter PFS than low $\mathrm{CD} 163^{+}$TAM density, with a pooled HR of 1.66 (95\% CI, 1.22-2.27). No significant heterogeneity was found across the studies $\left(I^{2}=12.6 \%\right.$, $P=0.11$; Fig. 3 ). Notably, only one study provided 
Table 1 Characteristics of studies included in the meta-analysis

\begin{tabular}{|c|c|c|c|c|c|c|c|c|c|c|c|}
\hline Study, Year & Country & $\begin{array}{l}\text { Number } \\
\text { of } \\
\text { subjects }\end{array}$ & Stage & $\begin{array}{l}\text { Treatment, } \\
\mathrm{n}(\%)\end{array}$ & $\begin{array}{l}\text { WHO } \\
\text { subtypes, } \\
n(\%)\end{array}$ & $\begin{array}{l}\text { Hodgkin-Reed- } \\
\text { Sternberg } \\
\text { EBV status, n (\%) }\end{array}$ & $\begin{array}{l}\text { Antibodies } \\
\text { (clone) }\end{array}$ & Scoring & Threshold(s) & $\begin{array}{l}\text { Follow-up median } \\
\text { (range) \& mean } \\
\text { (range) (in years) }\end{array}$ & $\begin{array}{l}\text { Outcome } \\
\text { correlation }\end{array}$ \\
\hline $\begin{array}{l}\text { Steidl et al., } \\
2010 \text { [10] }\end{array}$ & Canada & 166 & $\begin{array}{l}\text { Limited/ } \\
\text { advanced }\end{array}$ & $\begin{array}{l}\text { ABVD } \pm R T 165 \\
\text { (99), RT alone } 1 \\
\text { (1) }\end{array}$ & $\begin{array}{l}\text { NS } 140(84), \\
\text { MC } 11 \text { (7), } \\
\text { Others } 5 \text { (3), } \\
\text { NOS } 10(6)\end{array}$ & $\begin{array}{l}\text { Neg } 137(84) \\
\text { Pos } 27(16)\end{array}$ & CD68 (KP1) & Visual estimation & $5 \%, 25 \%$ & $4.0(0.5-20.8)$ & $\begin{array}{l}\text { CD68, adverse } \\
\text { (PFS, DSS) }\end{array}$ \\
\hline $\begin{array}{l}\text { Tzankov et al., } \\
2010 \text { [11] }\end{array}$ & Switzerland & 105 & $\begin{array}{l}\text { Limited/ } \\
\text { advanced }\end{array}$ & $\begin{array}{l}\mathrm{ABVD} \pm \mathrm{RT} 30 \\
(28), \mathrm{COPP} \pm \mathrm{RT} \\
48(46), \mathrm{RT} \text { alone } \\
27(26)\end{array}$ & $\begin{array}{l}\text { NS } 60(57), \\
\text { MC } 32(30) \\
\text { Others } 5(5), \\
\text { NOS } 8 \text { (8) }\end{array}$ & $\begin{array}{l}\text { Neg } 85(81), \\
\text { Pos } 20 \text { (19) }\end{array}$ & CD68 (PGM1) & Visual, cell counting & $0.82 \%$ & $11.8(1.0-27.6)$ & $\begin{array}{l}\text { CD68, adverse } \\
\text { (OS) }\end{array}$ \\
\hline $\begin{array}{l}\text { Kamper et al., } \\
2011 \text { [12] }\end{array}$ & Denmark & 288 & $\begin{array}{l}\text { Limited/ } \\
\text { advanced }\end{array}$ & $\begin{array}{l}\text { ABVD/COPP } \\
\pm R T, A B V D \\
\pm R T, R T \text { alone }\end{array}$ & $\begin{array}{l}\text { NS } 237(82), \\
\text { MC } 47(16), \\
\text { NOS } 4(1)\end{array}$ & $\begin{array}{l}\text { Neg } 193(67), \\
\text { Pos } 95 \text { (33) }\end{array}$ & $\begin{array}{l}\text { CD68 (KP1), } \\
\text { CD163 (10D6) }\end{array}$ & $\begin{array}{l}\text { Computer-assisted } \\
\text { point counting }\end{array}$ & $\begin{array}{l}7.8 \% \text { (CD68), } \\
21.1 \%(C D 163)\end{array}$ & $7.0(0.2-18.6)$ & $\begin{array}{l}\text { CD68, adverse } \\
\text { (EFS, OS); } \\
\text { CD163, adverse } \\
\text { (EFS, OS) }\end{array}$ \\
\hline $\begin{array}{l}\text { Hohaus et al., } \\
2011 \text { [13] }\end{array}$ & Italy & 93 & $\begin{array}{l}\text { Limited/ } \\
\text { advanced }\end{array}$ & $\begin{array}{l}\text { ABVD } \pm \text { RT } 55 \\
(59), \text { BEACOPP } \\
\pm \text { RT } 32 \text { (34), } \\
\text { MOPP/Other } \\
\pm \text { RT } 6 \text { (7) }\end{array}$ & $\begin{array}{l}\text { NS } 64(69), \\
\text { MC } 4(4), \\
\text { Other } 6 \text { (8), } \\
\text { NOS } 19 \text { (20) }\end{array}$ & $\begin{array}{l}\text { Neg } 37(69), \\
\text { Pos } 17(31)\end{array}$ & CD68 (PGM1) & Visual estimation & $5 \%$ & $1.1(0.1-6.8)$ & $\begin{array}{l}\text { CD68, adverse } \\
\text { (PFS) }\end{array}$ \\
\hline $\begin{array}{l}\text { Zaki et al., } \\
2011 \text { [23] }\end{array}$ & Japan & 82 & $\begin{array}{l}\text { Limited/ } \\
\text { advanced }\end{array}$ & $\begin{array}{l}\text { ABVD } \pm R T \\
\text { RT alone }\end{array}$ & $\begin{array}{l}\text { NS } 20(24) \\
\text { MC } 52(63) \\
\text { Other } 10(12)\end{array}$ & $N R$ & $\begin{array}{l}\text { CD68 (PGM1), } \\
\text { CD163 (10D6) }\end{array}$ & $\begin{array}{l}\text { Visual, cell counting } \\
\text { in } 0.146 \mathrm{~mm}^{2}\end{array}$ & $\begin{array}{l}\text { Median (CD68), } \\
\text { Median (CD163) }\end{array}$ & Mean: 4.0 (0.8-9.6) & $\begin{array}{l}\text { CD68, NSS (OS); } \\
\text { CD163, adverse } \\
\text { (OS) }\end{array}$ \\
\hline $\begin{array}{l}\text { Azambuja et al., } \\
2012 \text { [24] }\end{array}$ & USA & 265 & $\begin{array}{l}\text { Limited/ } \\
\text { advanced }\end{array}$ & $\begin{array}{l}A B V D \pm R T 265 \\
(100)\end{array}$ & $\begin{array}{l}\text { NS } 180(68) \\
\text { MC } 52(20) \\
\text { Others } 18(6) \\
\text { NOS } 15(6)\end{array}$ & $\begin{array}{l}\text { Neg } 122(58) \\
\text { Pos } 87(42)\end{array}$ & $\begin{array}{l}\text { CD68 (KP1), } \\
\text { CD163 (10D6) }\end{array}$ & Visual estimation & $\begin{array}{l}5 \%, 25 \%(C D 68), \\
5 \%, 25 \% \\
\text { (CD163) }\end{array}$ & $6(1.5-11.7)$ & $\begin{array}{l}\text { CD68, NSS } \\
\text { (PFS, DSS); CD163, } \\
\text { NSS (PFS, DSS) }\end{array}$ \\
\hline $\begin{array}{l}\text { Yoon et al., } \\
2012 \text { [14] }\end{array}$ & $\begin{array}{l}\text { South } \\
\text { Korea }\end{array}$ & 144 & $\begin{array}{l}\text { Limited/ } \\
\text { advanced }\end{array}$ & $\begin{array}{l}\text { ABVD } 113(79) \\
\text { C-MOPP } 10(7) \\
\text { ABVD/C-MOPP } \\
\text { hybrid } 15(10) \\
\text { BEACOPP } 6 \text { (4) }\end{array}$ & $\begin{array}{l}\text { NS } 90(63) \\
\text { MC } 34(24) \\
\text { Others } 11(7) \\
\text { NOS } 9(6)\end{array}$ & $\begin{array}{l}\text { Neg } 66(46), \\
\text { Pos } 78(54)\end{array}$ & $\begin{array}{l}\text { CD68 (KP1), } \\
\text { CD163 (10D6) }\end{array}$ & Visual estimation & $\begin{array}{l}20 \% \text { (CD68), } \\
20 \% \text { (CD163) }\end{array}$ & $5.4(0.7-19.0)$ & $\begin{array}{l}\text { CD68, adverse } \\
\text { (EFS, OS); CD163, } \\
\text { adverse (EFS, OS) }\end{array}$ \\
\hline $\begin{array}{l}\text { Tan et al., } \\
2012 \text { [15] }\end{array}$ & Canada & 287 & Advanced & $\begin{array}{l}\text { ABVD } \pm R T 144 \\
\text { (50), Stanford } \\
V \pm R T 143(50)\end{array}$ & $\begin{array}{l}\text { NS } 223(78), \\
\text { MC } 38(13) \\
\text { Others } 9(3), \\
\text { NOS 17(6) }\end{array}$ & $\begin{array}{l}\text { Neg } 238(83) \\
\text { Pos } 49(17)\end{array}$ & $\begin{array}{l}\text { CD68 (KP1), } \\
\text { CD163 (10D6) }\end{array}$ & $\begin{array}{l}\text { Computer-assisted } \\
\text { image analysis }\end{array}$ & $\begin{array}{l}12.7 \% \text { (CD68), } \\
16.8 \% \text { (CD163) }\end{array}$ & 5.5 & $\begin{array}{l}\text { CD63, adverse } \\
\text { (FFS, OS); CD163, } \\
\text { adverse (FFS, OS) }\end{array}$ \\
\hline $\begin{array}{l}\text { Sanchez- } \\
\text { Espiridion } \\
\text { et al., } \\
2012 \text { [31] }\end{array}$ & USA & 103 & Advanced & NR & $\begin{array}{l}\text { NS } 74(73), \\
\text { MC } 22(22) \\
\text { Others } 6(6)\end{array}$ & $N R$ & $\begin{array}{l}\text { CD68 (KP1 } \\
\text { and PGM1), } \\
\text { CD163 (10D6) }\end{array}$ & $\begin{array}{l}\text { Computer-assisted } \\
\text { point counting }\end{array}$ & $\begin{array}{l}5 \%, 25 \% \\
\text { median (CD68), } \\
5 \%, 25 \% \\
\text { median (CD163) }\end{array}$ & NR & $\begin{array}{l}\text { CD68, NSS } \\
\text { (FFS, OS); CD163, } \\
\text { NSS (FFS, OS) }\end{array}$ \\
\hline $\begin{array}{l}\text { Abdou et al., } \\
2013 \text { [16] }\end{array}$ & Egypt & 61 & $\begin{array}{l}\text { Limited/ } \\
\text { advanced }\end{array}$ & Chemotherapy & $\begin{array}{l}\text { NS } 33(54), \\
\text { MC } 20 \text { (33), } \\
\text { Others } 8 \text { (13) }\end{array}$ & NR & CD68 (KP1) & Visual, cell counting & $40 \%$ (CD68) & Mean: $1.2 \pm 1.7$ & $\begin{array}{l}\text { CD68, adverse } \\
\text { (OS) }\end{array}$ \\
\hline $\begin{array}{l}\text { Greaves et al., } \\
2013 \text { [17] }\end{array}$ & $\begin{array}{l}\text { United } \\
\text { Kingdom }\end{array}$ & 122 & $\begin{array}{l}\text { Limited/ } \\
\text { advanced }\end{array}$ & $\begin{array}{l}\text { Anthracycline-based } \\
\pm \text { RT } 56 \text { (46), } \\
\text { Alkalator-based } \pm \text { RT }\end{array}$ & $\begin{array}{l}\text { NS } 93(78) \\
\text { MC } 25(20) \\
\text { Others } 2(2)\end{array}$ & $\begin{array}{l}\text { Neg } 84(69), \\
\text { Pos } 38 \text { (31) }\end{array}$ & CD68 (KP1) & $\begin{array}{l}\text { Computer-assisted } \\
\text { image analysis }\end{array}$ & $5 \%, 15 \%$ & $16.5(2-40)$ & $\begin{array}{l}\text { CD68, adverse } \\
\text { (FFTF, OS) across }\end{array}$ \\
\hline
\end{tabular}


Table 1 Characteristics of studies included in the meta-analysis (Continued)

\begin{tabular}{|c|c|c|c|c|c|c|c|c|c|c|c|}
\hline & & & & $\begin{array}{l}52(43), \text { RT alone } \\
14(11)\end{array}$ & & & & & & & $\begin{array}{l}\text { the } 3 \text { defined } \\
\text { groups }\end{array}$ \\
\hline $\begin{array}{l}\text { Deau et al., } \\
2013 \text { [25] }\end{array}$ & France & 59 & $\begin{array}{l}\text { Limited/ } \\
\text { advanced }\end{array}$ & $\begin{array}{l}\text { ABVD } 47 \text { (80), EBVP } \\
8 \text { (14), BEACOPP } \\
3 \text { (5) }\end{array}$ & $\begin{array}{l}\text { NS } 54 \text { (92), } \\
\text { Non-NS } 5 \text { (8) }\end{array}$ & NR & CD68 (KP1) & Visual estimation & $25 \%$ & NR & $\begin{array}{l}\text { CD68, adverse } \\
\text { (PFS); CD68, } \\
\text { NSS (OS) }\end{array}$ \\
\hline $\begin{array}{l}\text { Panico et al., } \\
2015 \text { [26] }\end{array}$ & Italy & 121 & $\begin{array}{l}\text { Limited/ } \\
\text { advanced }\end{array}$ & $\begin{array}{l}\text { ABVD } \pm \text { RT } 101 \text { (83), } \\
\text { ABVD-like } \pm R T \\
20(17)\end{array}$ & $\begin{array}{l}\text { NS } 73(60) \\
\text { MC } 40(33) \\
\text { Others } 8(7)\end{array}$ & NR & CD68 (KP1) & Visual, cell counting & $\begin{array}{l}30 \text { per } \\
0.023 \mathrm{~mm}^{2}\end{array}$ & Mean: 3.5 (0.1-9.3) & $\begin{array}{l}\text { CD68, adverse } \\
\text { (OS); CD68, } \\
\text { NSS(PFS) }\end{array}$ \\
\hline $\begin{array}{l}\text { Casulo et al., } \\
2013 \text { [18] }\end{array}$ & USA & 81 & $\begin{array}{l}\text { Limited/ } \\
\text { advanced }\end{array}$ & $\begin{array}{l}\text { ABVD } 38(47), \\
\text { Stanford V } 11(13), \\
\text { ABVD/MOPP } 16 \\
\text { (20), others } 16(20)\end{array}$ & NR & NR & CD68 (KP1) & $\begin{array}{l}\text { Computer-assisted } \\
\text { image analysis }\end{array}$ & $30 \%$ & 8.8 & $\begin{array}{l}\text { CD68, adverse } \\
\text { (OS) }\end{array}$ \\
\hline $\begin{array}{l}\text { Koh et al., } \\
2014 \text { [19] }\end{array}$ & $\begin{array}{l}\text { South } \\
\text { Korea }\end{array}$ & 116 & $\begin{array}{l}\text { Limited/ } \\
\text { advanced }\end{array}$ & $\begin{array}{l}A B V D \pm R T 116 \\
(100)\end{array}$ & $\begin{array}{l}\text { NS } 78(67) \\
\text { MC } 22(19) \\
\text { Others } 8 \text { (7), } \\
\text { NOS } 8 \text { (7) }\end{array}$ & $\begin{array}{l}\text { Neg } 73(63) \\
\text { Pos } 43 \text { (37) }\end{array}$ & $\begin{array}{l}\text { CD68 (KP1), } \\
\text { CD163 (10D6) }\end{array}$ & Visual estimation & $\begin{array}{l}20 \% \text { (CD68), } \\
20 \% \text { (CD163) }\end{array}$ & $6.2(3.8-10.3)$ & $\begin{array}{l}\text { CD68, adverse } \\
\text { (EFS, DSS, OS); } \\
\text { CD163, adverse } \\
\text { (EFS, DSS, OS) }\end{array}$ \\
\hline $\begin{array}{l}\text { Ping et al., } \\
2014 \text { [27] }\end{array}$ & China & 72 & $\begin{array}{l}\text { Limited/ } \\
\text { advanced }\end{array}$ & $\begin{array}{l}\text { ABVD 49(68), } \\
\text { BEACOPP 13(18), } \\
\text { others } 10(14)\end{array}$ & $\begin{array}{l}\text { NS } 41(57), \\
\text { MC } 23 \text { (32), } \\
\text { Others } 8 \text { (11) }\end{array}$ & NR & CD68 (KP1) & Visual estimation & 250/HPF & $3.9(0.7-15)$ & CD68, NSS (OS); \\
\hline $\begin{array}{l}\text { Klein et al., } \\
2014 \text { [28] }\end{array}$ & USA & 88 & $\begin{array}{l}\text { Limited/ } \\
\text { advanced }\end{array}$ & $A B V D \pm R T ~ 88$ (100) & $\begin{array}{l}\text { NS } 55 \text { (63), } \\
\text { MC } 6 \text { (7), } \\
\text { Others } 1 \text { (1), } \\
\text { NOS } 19 \text { (22) }\end{array}$ & NR & $\begin{array}{l}\text { CD68 (KP1), } \\
\text { CD163 (10D6) }\end{array}$ & Visual estimation & $\begin{array}{l}5 \%, 25 \% \\
\text { (CD68), } 5 \% \\
25 \%(C D 163)\end{array}$ & NR & $\begin{array}{l}\text { CD68, NSS (OS); } \\
\text { CD163, adverse } \\
\text { (OS) } 25 \% \\
\text { threshold }\end{array}$ \\
\hline $\begin{array}{l}\text { Touati et al., } \\
2014 \text { [20] }\end{array}$ & France & 158 & $\begin{array}{l}\text { Limited/ } \\
\text { advanced }\end{array}$ & $\begin{array}{l}\text { ABVD 102(65), } \\
\text { ABVD/MOPP } 26 \\
\text { (17), BEACOPP } 11 \\
\text { (7), ABVD-like } 9 \text { (5), } \\
\text { others } 10 \text { (6) }\end{array}$ & $\begin{array}{l}\text { NS } 130(82), \\
\text { MC } 21(13), \\
\text { Others } 7 \text { (5) }\end{array}$ & $\begin{array}{l}\text { Neg } 106(67), \\
\text { Pos } 40(25), \\
\text { Not done } 12 \\
\text { (8) }\end{array}$ & CD68 (PGM1) & Visual estimation & $25 \%$ & $5.5(0.2-16.2)$ & $\begin{array}{l}\text { CD68, adverse } \\
\text { (PFS, OS) }\end{array}$ \\
\hline $\begin{array}{l}\text { Kayal et al., } \\
2014 \text { [30] }\end{array}$ & India & 100 & $\begin{array}{l}\text { Limited/ } \\
\text { advanced }\end{array}$ & $\begin{array}{l}A B V D \pm R T 88 \text { (88), } \\
\text { EVAP RT } 11(11), \\
\text { Other } 1 \text { (1) }\end{array}$ & $\begin{array}{l}\text { NS } 51 \text { (51), } \\
\text { MC } 47(47) \\
\text { Others } 2 \text { (2) }\end{array}$ & NR & $\begin{array}{l}\text { CD68 (CD68/ } \\
\text { G2) }\end{array}$ & Visual, cell counting & $\begin{array}{l}12.9 \%, \\
18.2 \%, 25 \% \\
\text { (the quartiles) }\end{array}$ & 5.7 & $\begin{array}{l}\text { CD68, NSS } \\
\text { (PFS, DSS) }\end{array}$ \\
\hline $\begin{array}{l}\text { Agur et al., } \\
2015 \text { [29] }\end{array}$ & Israel & 98 & $\begin{array}{l}\text { Limited/ } \\
\text { advanced }\end{array}$ & $\begin{array}{l}\text { ABVD 60(60), } \\
\text { BEACOPP 29(29), } \\
\text { others } 9 \text { (1) }\end{array}$ & $\begin{array}{l}\text { NS } 33(34) \\
\text { MC } 7 \text { (7), } \\
\text { Others } 58 \text { (59) }\end{array}$ & NR & CD68 (PGM1) & Visual, cell counting & $25 \%$ & Mean: 3.8 (0.9-7.8) & CD68, NSS (PFS) \\
\hline $\begin{array}{l}\text { Moreno et al., } \\
2015 \text { [21] }\end{array}$ & Spain & 249 & Advanced & NR & $\begin{array}{l}\text { NS } 162(65), \\
\text { MC } 68 \text { (27), } \\
\text { Others } 19 \text { (8) }\end{array}$ & NR & $\begin{array}{l}\text { CD68 (PGM1), } \\
\text { CD163 (10D6) }\end{array}$ & $\begin{array}{l}\text { Computer-assisted } \\
\text { point counting }\end{array}$ & $30 \%$ & NR & $\begin{array}{l}\text { CD68, adverse } \\
\text { (OS) }\end{array}$ \\
\hline $\begin{array}{l}\text { Jakovic et al., } \\
2016 \text { [22] }\end{array}$ & Serbia & 101 & Advanced & $\begin{array}{l}\text { ABVD } \pm \text { RT } 101 \\
(100)\end{array}$ & $\begin{array}{l}\text { NS } 80(79) \\
\text { MC } 13(13) \\
\text { Others } 8(8)\end{array}$ & NR & CD68 (PGM1) & Visual estimation & $25 \%$ & $8.6(0.2-16)$ & $\begin{array}{l}\text { CD68, adverse } \\
\text { (EFS, OS) }\end{array}$ \\
\hline
\end{tabular}

$A B V D$ doxorubicin, bleomycin, vinblastine, and dacarbazine, BEACOPP bleomycin, etoposide, doxorubicin, cyclophosphamide, vincristine, procarbazine, and prednisone, COPP cyclophosphamide, vincristine, procarbazine, and prednisone, C-MOPP cyclophosphamide, vincristine, procarbazine, prednisone, EVAP etoposide, vinblastine, adriamycin and prednisolone, EBVP epirubicin, bleomycin, vinblastine and prednisone, MOPP mustargen, oncovin, procarbazine, and prednisone, $R T$ radiotherapy, Stanford V vinblastine, doxorubicin, vincristine, bleomycin, mustard, etoposide, and prednisone, NS nodular sclerosis, MC mixed cellularity, Neg negative, POS positive, RT radiotherapy, NOS not otherwise specified, PFS progression-free survival, DSS disease-specific survival, EFS event free survival, OS overall survival, FFS failure free survival, FFTF frilarity, Neg negative, Pos positive, RT radiotherapy, NOS not otherwise specified, PFS progression-free survival, DSS from treatment failure, NR not reported, NSS not statistically significant, HPF high power field 
Table 2 Assessment of the risk of bias in each cohort study using the Newcastle-Ottawa scale

\begin{tabular}{|c|c|c|c|c|c|c|c|c|c|c|}
\hline \multirow[t]{2}{*}{ Study } & \multirow[b]{2}{*}{ REC } & \multicolumn{2}{|c|}{ Selection (0-4) } & \multirow[b]{2}{*}{ DO } & \multicolumn{2}{|c|}{ Comparability (0-2) } & \multicolumn{3}{|c|}{ Outcome (0-3) } & \multirow[t]{2}{*}{ Tota } \\
\hline & & SNEC & $\mathrm{AE}$ & & SC & AF & $\mathrm{AO}$ & $\mathrm{FU}$ & AFU & \\
\hline Steidl et al. [10] & 1 & 1 & 1 & 1 & 1 & 1 & 1 & 1 & 1 & 9 \\
\hline Tzankov et al. [11] & 1 & 1 & 1 & 1 & 0 & 0 & 0 & 1 & 1 & 6 \\
\hline Kamper et al. [12] & 1 & 1 & 1 & 1 & 1 & 1 & 1 & 1 & 0 & 8 \\
\hline Hohaus et al. [13] & 1 & 1 & 1 & 1 & 0 & 0 & 1 & 0 & 0 & 5 \\
\hline Zaki et al. [23] & 0 & 1 & 1 & 1 & 0 & 0 & 0 & 1 & 0 & 4 \\
\hline Azambuja et al. [24] & 1 & 1 & 1 & 1 & 1 & 1 & 1 & 1 & 0 & 8 \\
\hline Yoon et al. [14] & 1 & 1 & 1 & 1 & 1 & 0 & 1 & 1 & 1 & 8 \\
\hline Tan et al. [15] & 1 & 1 & 1 & 1 & 1 & 1 & 1 & 1 & 1 & 9 \\
\hline Sanchez-Espiridion et al. [31] & 0 & 1 & 1 & 1 & 0 & 0 & 0 & 1 & 0 & 4 \\
\hline Abdou et al. [16] & 0 & 1 & 1 & 1 & 0 & 0 & 0 & 0 & 0 & 3 \\
\hline Greaves et al. [17] & 1 & 1 & 1 & 1 & 1 & 1 & 1 & 1 & 1 & 9 \\
\hline Deau et al. [25] & 0 & 1 & 1 & 1 & 0 & 0 & 0 & 1 & 0 & 4 \\
\hline Panico et al. [26] & 1 & 1 & 1 & 1 & 0 & 0 & 1 & 1 & 0 & 6 \\
\hline Casulo et al. [18] & 1 & 1 & 1 & 1 & 1 & 0 & 1 & 1 & 0 & 7 \\
\hline Koh et al. [19] & 1 & 1 & 1 & 1 & 0 & 0 & 1 & 1 & 0 & 6 \\
\hline Ping et al. [27] & 0 & 1 & 1 & 1 & 0 & 0 & 1 & 1 & 0 & 5 \\
\hline Klein et al. [28] & 0 & 1 & 1 & 1 & 0 & 0 & 1 & 1 & 1 & 6 \\
\hline Touati et al. [20] & 1 & 1 & 1 & 1 & 0 & 0 & 0 & 1 & 1 & 6 \\
\hline Kayal et al. [30] & 1 & 1 & 1 & 1 & 0 & 0 & 1 & 1 & 0 & 6 \\
\hline Agur et al. [29] & 1 & 1 & 1 & 1 & 0 & 0 & 1 & 1 & 1 & 7 \\
\hline Moreno et al. [21] & 0 & 1 & 1 & 1 & 0 & 0 & 1 & 1 & 0 & 5 \\
\hline Jakovic et al. [22] & 1 & 1 & 1 & 1 & 0 & 1 & 0 & 1 & 0 & 6 \\
\hline
\end{tabular}

"1" indicates that the study has satisfied the item and " 0 " indications the opposite

$R E C$ representativeness of the exposed cohort, SNEC selection of the non-exposed cohort, $A E$ ascertainment of exposure, DO demonstration that outcome of interest was not present at start of study, SC study controls for age, sex, $A F$ study controls for any additional factors (Chemotherapy, radiotherapy), $A O$ assessment of outcome, FU follow-up long enough ( $36 \mathrm{M})$ for outcomes to occur, AFU adequacy of follow-up of cohorts ( $\geq 90 \%)$

relevant data on the correlation of $\mathrm{CD}_{163^{+}}$TAMs with DSS; therefore, the pooled analysis could not be performed.

\section{$\mathrm{CD}^{+} 8^{+}$and $\mathrm{CD}_{163}{ }^{+}$TAMs and clinical features}

Meta-analysis of five studies showed a high $\mathrm{CD}^{+} 8^{+} \mathrm{TAM}$ density was associated with the presence of EBV, with a pooled OR of 3.13 (95\% CI, 2.02-4.84). There was an indication of slight heterogeneity across the studies, but it did not reach statistical significance $\left(I^{2}=37.8 \%, P=0.17\right)$. The results of meta-analysis of the four studies showed a correlation between high $\mathrm{CD}_{163^{+}}$TAM density and the presence of EBV. Because significant heterogeneity was found across the studies $\left(I^{2}=67.9 \%, P=0.03\right)$, a pooled OR of 2.88 (95\% CI, 1.55-5.34) was calculated on the basis of a random-effects model (Fig. 4a).

Ten studies reported data on $\mathrm{CD} 68^{+}$TAMs and Ann Arbor stage in adult cHL. Meta-analysis of these studies found a trend for a correlation between high $\mathrm{CD}^{+} 8^{+}$ TAM density and advanced stage, with a pooled OR of 1.25 (95\% CI, 0.93-1.67). No significant heterogeneity was found across the studies $\left(I^{2}=23.6 \%, P=0.23\right)$. Four studies reported data on $\mathrm{CD}_{163}{ }^{+}$TAMs and Ann Arbor stage in adult cHL. Meta-analysis of the four studies showed a trend for a correlation between high $\mathrm{CD}_{163}{ }^{+}$ TAM density and advanced stage, with a pooled OR of 1.19 (95\% CI, 0.86-1.63). No significant heterogeneity was found across the studies $\left(I^{2}=0.0 \%, \quad P=0.91\right.$; Fig. 4b).

Six studies reported data on $\mathrm{CD}^{+} 8^{+}$TAMs and Bsymptoms in adult cHL. The result of meta-analysis of the six studies showed a trend for a correlation between a high $\mathrm{CD} 8^{+}$TAM density and B-symptoms, with a pooled OR of 1.35 (95\% CI, 0.90-2.01). No significant heterogeneity was found across the studies $\left(I^{2}=23.7 \%, P=0.26\right)$. Two studies reported data on $\mathrm{CD}_{163}{ }^{+}$TAMs and Bsymptoms in cHL. The result of meta-analysis of the two studies showed a trend for a correlation between high CD $163^{+}$TAM density and B-symptoms, with a pooled OR of 2.19 (95\% CI, 0.96-5.03). No significant heterogeneity was found across the studies $\left(I^{2}=57.1 \%, P=0.12\right.$; Fig. 4c).

Five studies reported data on $\mathrm{CD} 68^{+}$TAMs and IPS in adult cHL. The result of meta-analysis of the five studies 


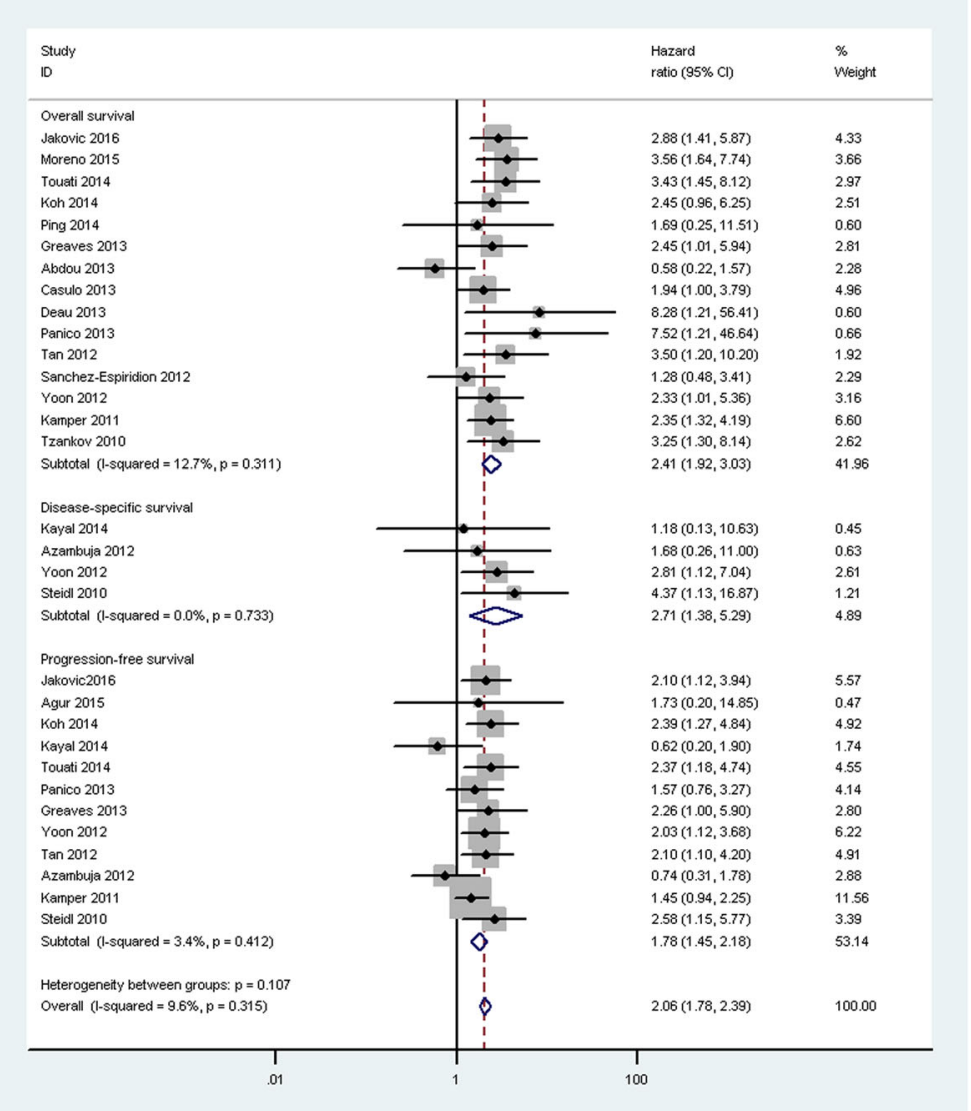

Fig. 2 Meta-analysis of the hazard ratios for progression-free survival, disease-specific survival, and overall survival for a high versus a low CD68 ${ }^{+}$ tumor-associated macrophage (TAM) density. Hazard ratios (HRs) and $95 \%$ confidence intervals (Cls) from individual studies are depicted as squares and horizontal lines, respectively. The pooled estimate is shown as a diamond shape, where the center represents the pooled HRs and the horizontal borders represent the $95 \% \mathrm{Cl}$. HRs are defined as high CD68 ${ }^{+}$versus low CD68 $8^{+}$TAM density; therefore, a hazard ratio > 1 represents a higher risk of death or progression associated with a high CD68 ${ }^{+}$TAM density

showed a trend correlation between a high $\mathrm{CD} 68^{+} \mathrm{TAM}$ density and higher IPS, with a pooled OR of 1.20 (95\% CI, 0.67-2.15). Three studies reported data on $\mathrm{CD}_{163}{ }^{+}$TAM density and IPS in adult cHL. The result of meta-analysis of the three studies showed a trend for a correlation between high $\mathrm{CD} 163^{+}$TAM density and a higher IPS, with a pooled OR of 2.00 (95\% CI, 0.92-4.35). Significant heterogeneity was found across the studies $\left(I^{2}=62.0 \%\right.$ for $\mathrm{CD}^{2} 8^{+}$TAMs and $I^{2}=72.5 \%$ for CD163 ${ }^{+}$TAMs, all $P<0.05$; Fig. $4 \mathrm{~d}$ ).

Five studies reported data on $\mathrm{CD} 68^{+}$TAMs and bulky disease in adult cHL; meta-analysis revealed a trend correlation between a high $\mathrm{CD} 68^{+}$TAM density and bulky disease, with a pooled OR of 1.47 (95\% CI, 0.88-2.47). Two studies reported data on $\mathrm{CD} 163^{+}$TAMs and bulky disease in adult cHL. The result of meta-analysis of the two studies showed a trend for a correlation between a high $\mathrm{CD}_{163}{ }^{+}$TAM density and bulky disease, with a pooled OR of 1.19 (95\% CI, 0.72-1.96). No significant heterogeneity was found across the studies $\left(I^{2}=40.4 \%\right.$ for
$\mathrm{CD}^{+} 8^{+}$TAM density and $I^{2}=0.0 \%$ for CD163 expression, all $P>0.10$; Fig. 4e).

\section{Publication bias}

A more formal evaluation of CD68 ${ }^{+}$TAMs using Begg's and Egger's tests showed no evidence of significant publication bias (OS, Begg's test $P=0.488$; Egger's test $P=0.522$; shown in Fig. 5a; PFS, Begg's test $P=0.732$; Egger's test $P=0.639$; shown in Fig. $5 \mathrm{~b}$ ). For $\mathrm{CD} 163^{+}$ TAMs, there was no evidence for significant publication bias (OS, Begg's test $P=0.230$; Egger's test $P=0.172$ (Fig. 5c); PFS, Begg's test $P=1.000$; Egger's test $P=0.356$ (Fig. 5d)).

\section{Discussion}

Many recent studies have focused on the impact of non-neoplastic cells on disease pathobiology, specifically immunohistochemical typing of cells in the microenvironment, with the goal of identifying potential prognostic markers and therapeutic targets in adult cHL. Due to 


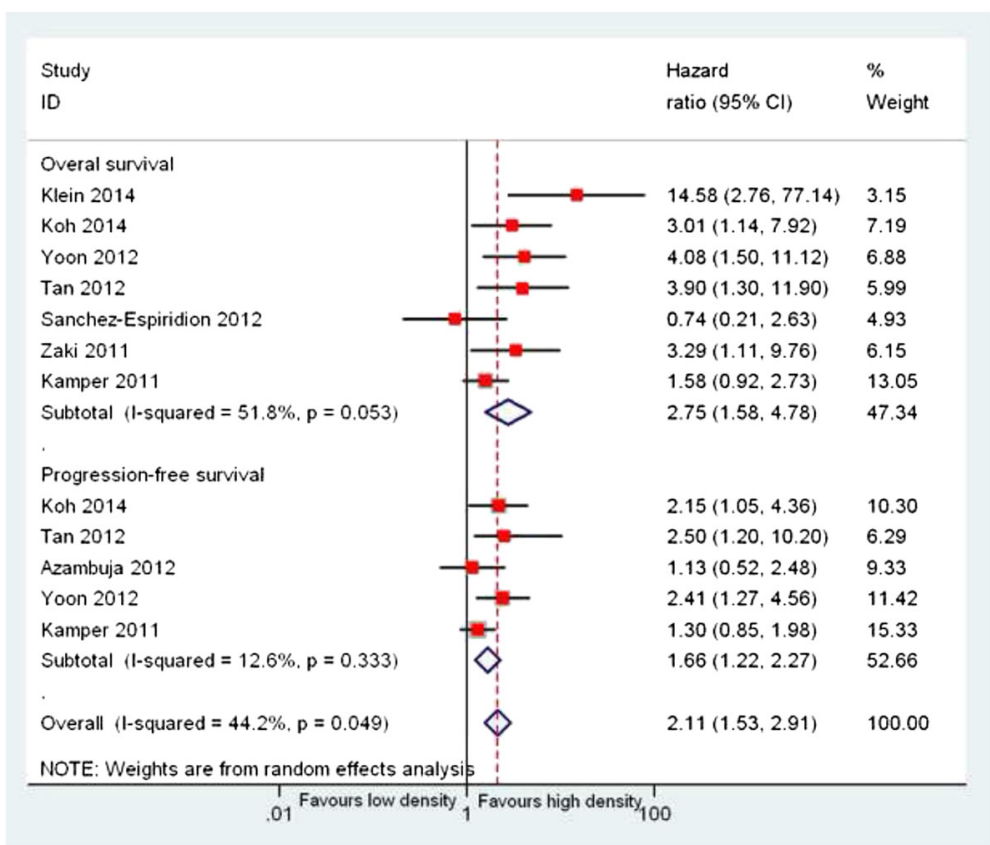

Fig. 3 Meta-analysis of the hazard ratios for progression-free survival and overall survival for high $\mathrm{CD}_{163^{+}}$versus low CD $163^{+}$tumor-associated macrophage (TAM) density. Hazard ratios and $95 \%$ confidence intervals for death or progression associated with high versus low CD163 ${ }^{+}$TAM density

a

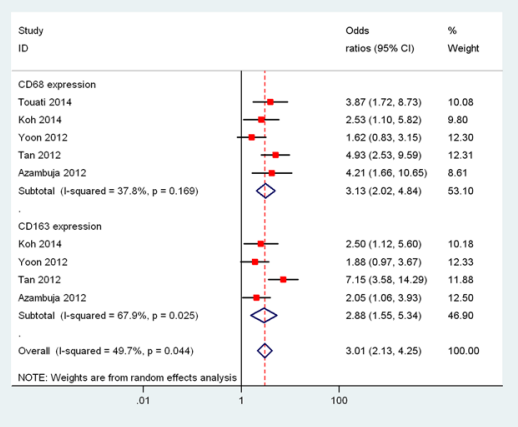

d

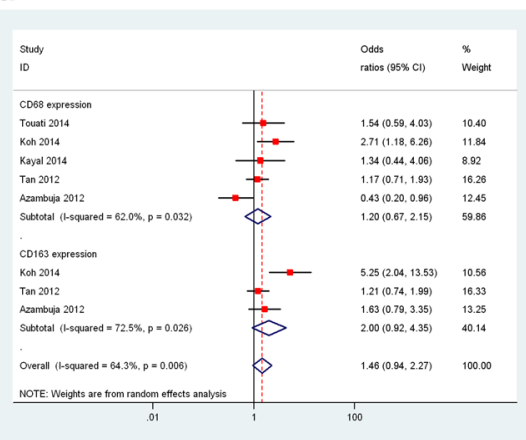

b

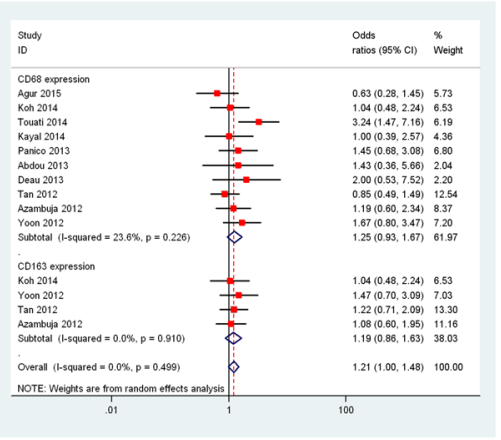

e

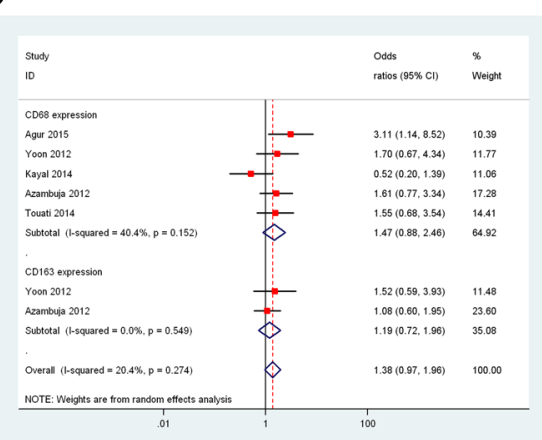

Fig. 4 Meta-analysis of the association between $\mathrm{CD}^{+} 8^{+}$and $\mathrm{CD} 163^{+}$tumor-associated macrophage (TAM) density and clinical features. The Forest plots show (a) the association between a high $\mathrm{CD}^{+} 8^{+}$and $\mathrm{CD} 163^{+}$TAM density and presence of Epstein-Barr virus of adult classical Hodgkin lymphoma (cHL); (b) the association between a high $\mathrm{CD}_{6} 8^{+}$and $\mathrm{CD} 163^{+} \mathrm{TAM}$ density and presence of Ann Arbor stage of adult $\mathrm{CHL}$; (c) the association between a high $\mathrm{CD}^{+} 8^{+}$and $\mathrm{CD} 163^{+}$TAM density and B-symptoms of adult $\mathrm{CHL} ;(\mathbf{d})$ the association between a high $\mathrm{CD} 68^{+}$and $\mathrm{CD}_{163^{+}} \mathrm{TAM}$ density and International Prognostic Factors Project Score of adult $\mathrm{CHL}$; and (e) the association between a high $\mathrm{CD} 68^{+}$and $\mathrm{CD} 163^{+} \mathrm{TAM}$ density and bulky disease of adult $\mathrm{CHL}$ 

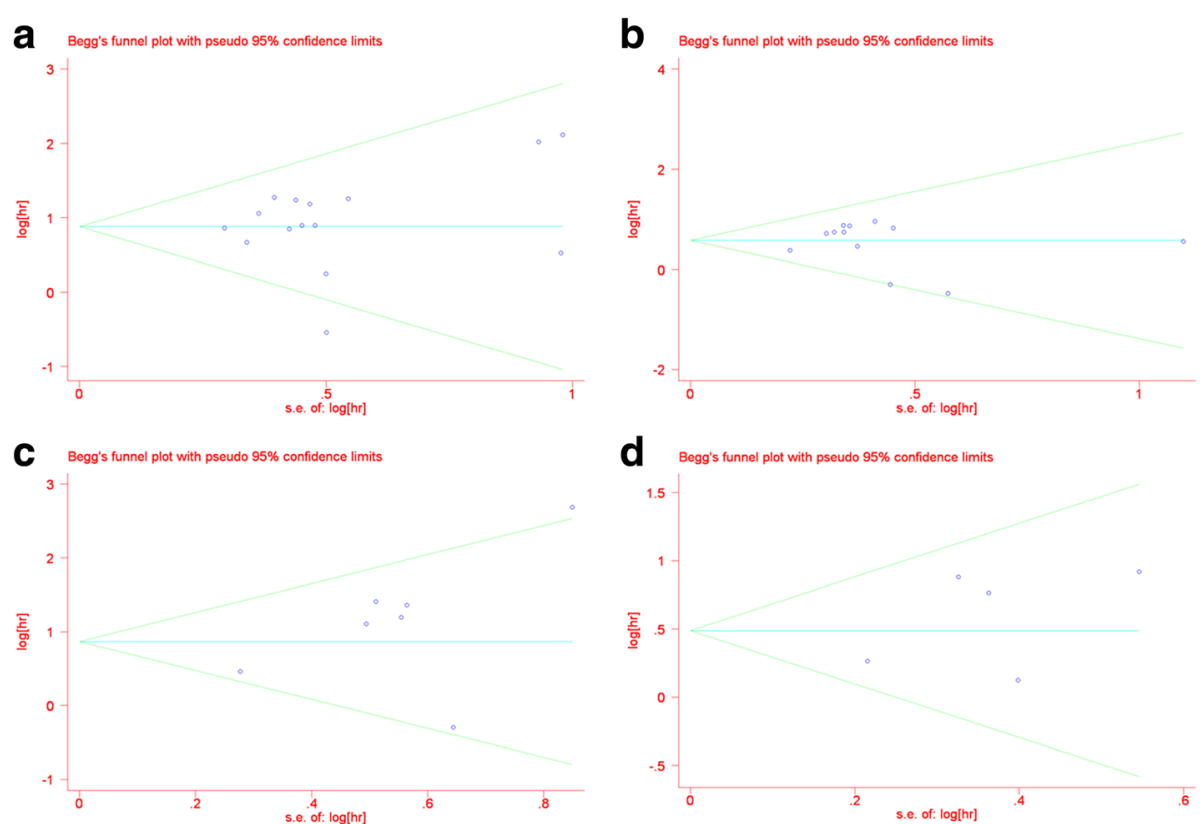

Fig. 5 Begg's funnel plot showed no publication bias among the included studies. a CD68 ${ }^{+}$tumor-associated macrophages (TAMs) and overall survival OS $(P=0.488)$. b CD68 $8^{+}$TAMs and PFS $(P=0.732)$. $\mathbf{c} C D 163^{+}$TAMs and OS $(P=0.230)$. d CD $163^{+}$TAMs and PFS $(P=1.000)$

flawed statistical analyses and an inability to validate findings, few biomarkers have translated into clinical practice. Meta-analysis is a valuable tool in biomarker validation; thus, in this study, we carried out a meta-analysis to examine the correlation between TAMs and adult cHL prognosis.

This is the first meta-analysis investigating survival of cHL patients in which the survival of patients with high and low density of $\mathrm{CD} 8^{+}$and $\mathrm{CD} 163^{+}$TAMs was compared. Our results demonstrated that a high density of either $\mathrm{CD}^{+} 8^{+}$or $\mathrm{CD}_{163}{ }^{+}$TAMs in the tumor microenvironment significantly predicted poor OS and shorter PFS for adult cHL $(P=0.000$ and $P=0.000$, respectively). A high $\mathrm{CD} 68^{+}$TAM density was associated with worse DSS than that in the group with a low $\mathrm{CD} 68^{+}$TAM density $(P=0.004)$. Furthermore, we also conducted a pooled analysis on the correlation between macrophageassociated markers and adult cHL EBV positivity. This finding suggests that a high density of either $\mathrm{CD} 68^{+}$or $\mathrm{CD}_{163^{+}}$TAMs is strongly correlated with EBV positivity ( $P=0.000$ and $P=0.001$, respectively). In addition, these results suggested a trend towards a high density of both $\mathrm{CD}_{6} 8^{+}$and $\mathrm{CD}_{163^{+}}$TAMs with the presence of B-symptoms, advanced stage, bulky disease, and an IPS greater than 3; however, these results were not statistically significant. Taken together, the results of our pooled analysis support that a higher density of either $\mathrm{CD} 68^{+}$or $\mathrm{CD}_{163^{+}}{ }^{\mathrm{TAM}} \mathrm{s}$ in the tumor microenvironment of adult $\mathrm{cHL}$ is associated with a higher risk of worst outcome.
The results of our meta-analysis indicate an association between increased density of both $\mathrm{CD}^{+} 8^{+}$and $\mathrm{CD}_{163}{ }^{+}$TAMs in the tumor microenvironment of adult $\mathrm{cHL}$ and poor outcome. Among the included studies, different score methods and threshold values were used in the measurement of $\mathrm{CD} 68^{+}$and $\mathrm{CD} 163^{+}$TAMs using immunohistochemistry. Most of the studies used manual visual scoring techniques, ranging from cell counting $[11,23,24]$ to computer-assisted methods of point counting $[12,21]$ and image analysis $[15,17]$. The lack of consistent and reproducible of thresholds in these studies made it difficult to separate patients into lowand high-risk populations. Reproducibility of data produced in different laboratories and of assay methods for tissue sections will need to be a focus of future studies. Few biomarkers have translated into clinical practice since the reproducibility of immunohistochemical scoring has been suggested as a reason for inconclusive results, and thus more robust multigene predictors have been reported based on expression profiling [10]. However, these gene expression studies were limited by small case numbers and the lack of available clinical data.

In addition, the antibody used to stain macrophages varied. It has been reported that the KP1 clone does not only react specifically with macrophages, but also reacts with myeloid and fibroblastic cells [39]. The clone 10D6 for CD163 is more specific for macrophages than either the KP1 or PGM1 clones for CD68 [40]. Thus, CD163 may be a better marker for TAMs than CD68. When 
antibodies to CD68 and CD163 have both been used, some studies have noted discrepancies in the association of macrophages with patient outcomes. Martin-Moreno et al. [21] observed an association between increased CD68-stained cells and DSS in a cohort, but no association was observed using an antibody against CD163. In contrast, Zaki et al. [23] and Klein et al. [28] only saw an association between CD163 and outcome. Some studies indicate that TAMs in adult cHL promote tumor growth and angiogenesis, suppression of adaptive immune responses, and contribute to immune evasion by tumor cells, and thus may be associated with poor prognosis [41-44].

These analyses have some advantages and important implications. First, study quality scores, assessed using the NOS, had a mean score of 6.23 , giving validity to the results of the present meta-analysis. Second, Egger's test did not detect publication bias, indicating that the results are not biased. Third, this study shows that a high density of both $\mathrm{CD} 68^{+}$and $\mathrm{CD} 163^{+}$TAMs is associated with poorer outcome, which suggests that the TAMs may be useful as a drug therapeutic target. Fourth, our study identifies a subgroup of adult cHL tumors with poorer outcome. Finally, it highlights the importance of the development of an accurate biomarker for assessment of adult cHL.

The meta-analysis performed in this study had several limitations. First, negative studies are less frequently published, or are published with less detailed results, making them less assessable, potentially leading to some bias. Second, our meta-analysis is based on data from trials from which the results have been published, and we did not obtain updated individual patient data. Use of individual patient data may further enhance the accuracy and reduce the uncertainty of the estimates. Third, because of the variety of endpoints reported in adult cHL studies, we operationally defined adult cHL PFS to include EFS or FFS in studies that did not provide PFS. Fourth, some of the HRs with $95 \%$ CIs were extracted from the Kaplan-Meier survival curve. Finally, among the included studies in the current metaanalysis, six had a follow-up time of less than 5 years, which may have incorporated bias.

\section{Conclusions}

In conclusion, the results presented here indicate that a high density of either $\mathrm{CD}^{+} 8^{+}$or $\mathrm{CD} 163^{+} \mathrm{TAMs}$ in the tumor microenvironment of adult $\mathrm{cHL}$ is associated with poor survival. A high density of both $\mathrm{CD} 68^{+}$and $\mathrm{CD} 163^{+}$ TAMs was associated with the presence of EBV in neoplastic cells, and might provide essential information for the prediction of advanced stage, B-symptoms, bulky disease, and higher IPS. Evaluation of TAMs should be considered in prospective clinical trials, and patients with increased TAMs may benefit from more intensive chemotherapy or novel agents designed to disrupt crosstalk between Hodgkin-Reed-Sternberg cells and benign macrophages.

\section{Acknowledgements \\ This work was supported by the National Natural Science Foundation of China (NO.81160298). The funders had no role in study design, data collection and analysis, decision to publish, or preparation of the manuscript.}

\section{Authors' contributions}

$\mathrm{BPG}, \mathrm{XHT}, \mathrm{QK}$, and $\mathrm{HC}$ conceived of the study, selected studies and extracted the data, analyzed and interpreted the data, wrote the first draft of the manuscript, and revised the manuscript. All authors read and approved the final manuscript.

\section{Competing interests}

The authors declare that they have no competing interests.

Received: 17 June 2016 Accepted: 4 October 2016

Published online: 17 October 2016

\section{References}

1. Engert A, Diehl V, Franklin J, Lohri A, Dorken B, Ludwig WD, et al. Escalated-dose BEACOPP in the treatment of patients with advanced-stage Hodgkin's lymphoma: 10 years of follow-up of the GHSG HD9 study. J Clin Oncol. 2009;27(27):4548-54.

2. Engert A, Plutschow A, Eich HT, Lohri A, Dorken B, Borchmann P, et al. Reduced treatment intensity in patients with early-stage Hodgkin's lymphoma. N Engl J Med. 2010;363(7):640-52.

3. Viviani S, Zinzani PL, Rambaldi A, Brusamolino E, Levis A, Bonfante V, et al. $A B V D$ versus BEACOPP for Hodgkin's lymphoma when high-dose salvage is planned. N Engl J Med. 2011;365(3):203-12.

4. Varterasian M, Ratanatharathorn V, Uberti JP, Karanes C, Abella E, Momin F, et al. Clinical course and outcome of patients with Hodgkin's disease who progress after autologous transplantation. Leuk Lymphoma. 1995;20(1-2):59-65.

5. Shamash J, Lee SM, Radford JA, Rohatiner AZ, Chang J, Morgenstern GR, et al. Patterns of relapse and subsequent management following high-dose chemotherapy with autologous haematopoietic support in relapsed or refractory Hodgkin's lymphoma: a two centre study. Ann Oncol. 2000;11(6):715-9.

6. Castellino SM, Geiger AM, Mertens AC, Leisenring WM, Tooze JA, Goodman P, et al. Morbidity and mortality in long-term survivors of Hodgkin lymphoma: a report from the Childhood Cancer Survivor Study. Blood. 2011:117(6):1806-16.

7. Moccia AA, Donaldson J, Chhanabhai M, Hoskins PJ, Klasa RJ, Savage KJ, et al. International Prognostic Score in advanced-stage Hodgkin's lymphoma: altered utility in the modern era. J Clin Oncol. 2012;30(27):3383-8.

8. Hasenclever D, Diehl V. A prognostic score for advanced Hodgkin's disease. International Prognostic Factors Project on Advanced Hodgkin's Disease. N Engl J Med. 1998;339(21):1506-14.

9. Steidl C, Connors JM, Gascoyne RD. Molecular pathogenesis of Hodgkin's lymphoma: increasing evidence of the importance of the microenvironment. J Clin Oncol. 2011;29(14):1812-26.

10. Steidl C, Lee T, Shah SP, Farinha P, Han G, Nayar T, et al. Tumor-associated macrophages and survival in classic Hodgkin's lymphoma. N Engl J Med. 2010;362(10):875-85

11. Tzankov A, Matter MS, Dirnhofer S. Refined prognostic role of CD68-positive tumor macrophages in the context of the cellular micromilieu of classical Hodgkin lymphoma. Pathobiology. 2010;77(6):301-8.

12. Kamper P, Bendix K, Hamilton-Dutoit S, Honore B, Nyengaard JR, d'Amore F. Tumor-infiltrating macrophages correlate with adverse prognosis and Epstein-Barr virus status in classical Hodgkin's lymphoma. Haematologica. 2011;96(2):269-76.

13. Hohaus S, Santangelo R, Giachelia M, Vannata B, Massini G, Cuccaro A, et al. The viral load of Epstein-Barr virus (EBV) DNA in peripheral blood predicts for biological and clinical characteristics in Hodgkin lymphoma. Clin Cancer Res. 2011;17(9):2885-92. 
14. Yoon DH, Koh YW, Kang HJ, Kim S, Park CS, Lee SW, et al. CD68 and CD163 as prognostic factors for Korean patients with Hodgkin lymphoma. Eur J Haematol. 2012;88(4):292-305.

15. Tan KL, Scott DW, Hong F, Kahl BS, Fisher RI, Bartlett NL, et al. Tumor-associated macrophages predict inferior outcomes in classic Hodgkin lymphoma: a correlative study from the E2496 Intergroup trial. Blood. 2012;120(16):3280-7

16. Abdou AG, Asaad NY, Loay I, Shabaan M, Badr N. The prognostic role of tumor-associated macrophages and dendritic cells in classic Hodgkin's Iymphoma. J Environ Pathol Toxicol Oncol. 2013;32(4):289-305.

17. Greaves P, Clear A, Coutinho R, Wilson A, Matthews J, Owen A, et al. Expression of FOXP3, CD68, and CD20 at diagnosis in the microenvironment of classical Hodgkin lymphoma is predictive of outcome. J Clin Oncol. 2013;31(2):256-62

18. Casulo C, Arcila M, Bohn OL, Teruya-Feldstein J, Maragulia J, Moskowitz CH. Tumor associated macrophages in relapsed and refractory Hodgkin lymphoma. Leuk Res. 2013;37(9):1178-83.

19. Koh YW, Park CS, Yoon DH, Suh C, Huh J. CD163 expression was associated with angiogenesis and shortened survival in patients with uniformly treated classical Hodgkin lymphoma. PLoS One. 2014;9(1):e87066.

20. Touati M, Delage-Corre M, Monteil J, Abraham J, Moreau S, Remenieras L, et al. CD68-positive tumor-associated macrophages predict unfavorable treatment outcomes in classical Hodgkin lymphoma in correlation with interim fluorodeoxyglucose-positron emission tomography assessment. Leuk Lymphoma. 2015;56(2):332-41.

21. Martin-Moreno AM, Roncador G, Maestre L, Mata E, Jimenez S, Martinez-Torrecuadrada JL, et al. CSF1R protein expression in reactive lymphoid tissues and lymphoma: its relevance in classical Hodgkin lymphoma. PLoS One. 2015;10(6):e0125203.

22. Jakovic LR, Mihaljevic BS, Andjelic BM, Bogdanovic AD, Perunicic Jovanovic $M D$, Babic DD, et al. Prognostic value of lymphocyte/monocyte ratio in advanced Hodgkin lymphoma: correlation with International Prognostic Score and tumor associated macrophages. Leuk Lymphoma. 2016;57(8):1839-47.

23. Zaki MA, Wada N, Ikeda J, Shibayama H, Hashimoto K, Yamagami T, et al. Prognostic implication of types of tumor-associated macrophages in Hodgkin lymphoma. Virchows Arch. 2011;459(4):361-6.

24. Azambuja D, Natkunam Y, Biasoli I, Lossos IS, Anderson MW, Morais JC, et al. Lack of association of tumor-associated macrophages with clinical outcome in patients with classical Hodgkin's lymphoma. Ann Oncol. 2012;23(3):736-42.

25. Deau B, Bachy E, Ribrag V, Delarue R, Rubio MT, Bosq J, et al. Macrophage, mast cell and $T$ lymphocyte infiltrations are independent predictive biomarkers of primary refractoriness or early relapse in classical Hodgkin lymphoma. Leuk Lymphoma. 2013;54(1):41-5.

26. Panico L, Tenneriello V, Ronconi F, Lepore M, Cantore N, Dell'Angelo AC, et al. High CD20+ background cells predict a favorable outcome in classical Hodgkin lymphoma and antagonize CD68+ macrophages. Leuk Lymphoma. 2015;56(6):1636-42.

27. Ping LY, Ding N, Shi YF, Sun L, Zheng W, Xie Y, et al. Clinical characteristics and prognosis analysis of patients with LMP-1 positive Hodgkin's lymphoma after EBV infection. J Exp Hematol. 2014;22(1):78-84.

28. Klein JL, Nguyen TT, Bien-Willner GA, Chen L, Foyil KV, Bartlett NL, et al. CD163 immunohistochemistry is superior to CD68 in predicting outcome in classical Hodgkin lymphoma. Am J Clin Pathol. 2014;141(3):381-7.

29. Agur A, Amir G, Paltiel O, Klein M, Dann EJ, Goldschmidt H, et al. CD68 staining correlates with the size of residual mass but not with survival in classical Hodgkin lymphoma. Leuk Lymphoma. 2015;56(5):1315-9.

30. Kayal S, Mathur S, Karak AK, Kumar L, Sharma A, Bakhshi S, et al. CD68 tumor-associated macrophage marker is not prognostic of clinical outcome in classical Hodgkin lymphoma. Leuk Lymphoma. 2014;55(5):1031-7.

31. Sánchez-Espiridión B, Martin-Moreno AM, Montalbán C, Medeiros LJ, Vega F, Younes A, et al. Immunohistochemical markers for tumor associated macrophages and survival in advanced classical Hodgkin's lymphoma. Haematologica. 2012;97(7):1080-4.

32. Liberati A, Altman DG, Tetzlaff J, Mulrow C, Gotzsche PC, loannidis JP, et al. The PRISMA statement for reporting systematic reviews and meta-analyses of studies that evaluate health care interventions: explanation and elaboration. PLoS Med. 2009;6(7):e1000100.

33. Stang A. Critical evaluation of the Newcastle-Ottawa scale for the assessment of the quality of nonrandomized studies in meta-analyses. Eur J Epidemiol. 2010;25(9):603-5.
34. Parmar MK, Torri V, Stewart L. Extracting summary statistics to perform meta-analyses of the published literature for survival endpoints. Stat Med. 1998;17(24):2815-34.

35. Higgins JP, Thompson SG. Quantifying heterogeneity in a meta-analysis. Stat Med. 2002;21(11):1539-58.

36. DerSimonian R, Laird N. Meta-analysis in clinical trials. Control Clin Trials. 1986;7(3):177-88.

37. Begg CB, Mazumdar M. Operating characteristics of a rank correlation test for publication bias. Biometrics. 1994;50(4):1088-101.

38. Egger M, Davey Smith G, Schneider M, Minder C. Bias in meta-analysis detected by a simple, graphical test. BMJ. 1997;315(7109):629-34.

39. Kunisch E, Fuhrmann R, Roth A, Winter R, Lungershausen W, Kinne RW. Macrophage specificity of three anti-CD68 monoclonal antibodies (KP1, EBM11, and PGM1) widely used for immunohistochemistry and flow cytometry. Ann Rheum Dis. 2004;63(7):774-84

40. Lau SK, Chu PG, Weiss LM. CD163: a specific marker of macrophages in paraffin-embedded tissue samples. Am J Clin Pathol. 2004;122(5):794-801.

41. Lawrence T, Natoli G. Transcriptional regulation of macrophage polarization: enabling diversity with identity. Nat Rev Immunol. 2011;11(11):750-61.

42. Mantovani A, Germano G, Marchesi F, Locatelli M, Biswas SK. Cancerpromoting tumor-associated macrophages: new vistas and open questions. Eur J Immunol. 2011:41(9):2522-5.

43. Murray PJ, Wynn TA. Protective and pathogenic functions of macrophage subsets. Nat Rev Immunol. 2011:11(11):723-37.

44. Allavena P, Mantovani A. Immunology in the clinic review series; focus on cancer: tumour-associated macrophages: undisputed stars of the inflammatory tumour microenvironment. Clin Exp Immunol. 2012;167(2):195-205.

\section{Submit your next manuscript to BioMed Central and we will help you at every step:}

- We accept pre-submission inquiries

- Our selector tool helps you to find the most relevant journal

- We provide round the clock customer support

- Convenient online submission

- Thorough peer review

- Inclusion in PubMed and all major indexing services

- Maximum visibility for your research

Submit your manuscript at www.biomedcentral.com/submit

) Biomed Central 